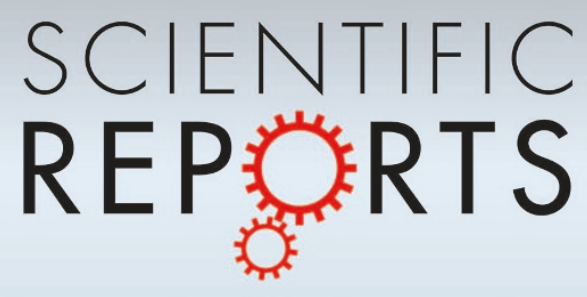

OPEN

SUBJECT AREAS:

APPLIED PHYSICS

ELECTRONICS, PHOTONICS AND DEVICE PHYSICS

Received

16 December 2013

Accepted

5 June 2014

Published

26 June 2014

Correspondence and requests for materials should be addressed to

S.M.W. (shumin@ mail.sim.ac.cn)

\section{InPBi Single Crystals Grown by Molecular Beam Epitaxy}

\author{
K. Wang' ', Y. Gu', H. F. Zhou' ', L. Y. Zhang' ', C. Z. Kang ${ }^{2}$, M. J. Wu ${ }^{3}$, W. W. Pan' ', P. F. Lu', Q. Gong' \\ \&S. M. Wang ${ }^{1,5}$
}

'State Key Laboratory of Functional Materials for Informatics, Shanghai Institute of Microsystem and Information Technology, CAS, 865 Changning Road, Shanghai 200050, China, ${ }^{2}$ Qufu Normal University, 57 West Jinxuan Road, Qufu 273165, China,

${ }^{3}$ Paul-Drude-Institut für Festkörperelektronik, Hausvogteiplatz 5-7, Berlin D-10 1 17, Germany, ${ }^{4}$ State Key Laboratory of Information Photonics and Optical Communications, Ministry of Education, Beijing University of Posts and Telecommunications, 10 West Tucheng Road, Beijing 100876, China, ${ }^{5}$ Department of Microtechnology and Nanoscience, Chalmers University of Technology, 41296 Gothenburg, Sweden.

InPBi was predicted to be the most robust infrared optoelectronic material but also the most difficult to synthesize within In-VBi ( $\mathrm{V}=\mathrm{P}, \mathrm{As}$ and $\mathrm{Sb}) 25$ years ago. We report the first successful growth of InPBi single crystals with $\mathrm{Bi}$ concentration far beyond the doping level by gas source molecular beam epitaxy. The InPBi thin films reveal excellent surface, structural and optical qualities making it a promising new III-V compound family member for heterostructures. The Bi concentration is found to be $2.4 \pm 0.4 \%$ with $94 \pm$ $5 \% \mathrm{Bi}$ atoms at substitutional sites. Optical absorption indicates a band gap of $1.23 \mathrm{eV}$ at room temperature while photoluminescence shows unexpectedly strong and broad light emission at 1.4-2.7 $\mu \mathrm{m}$ which can't be explained by the existing theory.

\footnotetext{
C ompound semiconductors play a pivotal role in modern optoelectronics whose energy gap and lattice constant can be tailored to meet various device requirements. While semiconductors containing N, P, As and $\mathrm{Sb}$ in the column $\mathrm{V}$ of the periodic table have been extensively investigated, bismuth (Bi) containing alloys are less explored. Isoelectronic impurities in host materials, such as $\mathrm{N}$ and $\mathrm{Bi}$ in $\mathrm{GaP}$ and $\mathrm{InP}$ at the doping level, reveal rich spectroscopic features near the band gap of the host material ${ }^{1-4}$. When the impurity concentration increases from the doping level to the dilute alloy or even the concentrated alloy level, interaction between the impurity energy level and the band edge of the host material results in strong band gap bowing effect in dilute nitrides ${ }^{5,6}$ and also in dilute $\mathrm{GaAsBi}^{7}$. This property, which can be well explained by the band anti-crossing model $^{8}$, has been employed not only to demonstrate uncooled high-speed telecom lasers on GaAs ${ }^{9}$ and the record high conversion efficiency of triple junction solar cells both using dilute nitrides ${ }^{10}$, but also to trigger great intention to search for relatively unexplored dilute bismides for band engineering. Berding et al. theoretically analyzed InSbBi, InAsBi and InPBi as potential candidates for mid- and far-infrared (IR) optoelectronics applications in 1988 and pointed out that InSbBi was the easiest and InPBi was the most difficult to mix, but once formed, InPBi was the hardest among the three materials ${ }^{11}$, making it an attractive narrow-gap material candidate. Since InBi crystallizes in the tetragonal $\mathrm{PbO}$-type structure rather than the cubic zinc-blende structure common for semiconducting III-V compounds, the mixing of InBi with other materials of zinc-blende structure may lead to problems ${ }^{12}$. Early work by Oe et al. ${ }^{13}$ and Noreika et al. ${ }^{14}$ showed a very narrow growth window for InSbBi due to the weak bonding of In-Bi and formation of metallic In or Bi droplets. InAsBi samples grown at low temperatures show binary InBi inclusions with a tetragonal $\mathrm{PbO}$ phase ${ }^{12}$. To our knowledge, InPBi single crystal has never been reported since the first theoretical prediction made 25 years ago ${ }^{11}$.

Here we report the first successful growth of $\operatorname{InP}_{1-{ }_{x}} \mathrm{Bi}_{\mathrm{x}}$ thin films with Bi concentration of $x=2.4 \pm 0.4 \%$ by gas source molecular beam epitaxy (MBE). The InPBi crystal not only reveals excellent material quality, but also exhibits strong and broad room temperature photoluminescence (PL) from deep levels at 1.4-2.7 $\mu \mathrm{m}$ which provokes new theory to account for this phenomenon.
}

\section{Results}

Surface and structural properties. A set of samples with thickness of $420 \mathrm{~nm}$ were grown by gas source MBE and their growth parameters are listed in Table 1. Details of the growth procedure can be found in Methods. Sample a, $\mathrm{b}$ and $\mathrm{c}$ have the same flux of all three elements but different growth temperatures. Atomic force microscopy 
Table 1 Growth parameters and RMS roughness values measured from AFM of the three $\mathrm{InPBi}$ samples grown at different growth temperatures

\begin{tabular}{lccccc} 
Sample No. & $\mathrm{T}_{\mathrm{g}}\left({ }^{\circ} \mathrm{C}\right)$ & $\mathrm{T}_{\mathrm{In}}\left({ }^{\circ} \mathrm{C}\right)$ & $\mathrm{T}_{\mathrm{Bi}}\left({ }^{\circ} \mathrm{C}\right)$ & $\mathrm{PH}_{3}$ (Torr) & $\mathrm{RMS}(\mathrm{nm})$ \\
\hline $\mathbf{a}$ & 364 & 940 & 465 & 350 & 84.6 \\
$\mathbf{b}$ & 324 & 940 & 465 & 350 & 0.93 \\
$\mathbf{c}$ & 275 & 940 & 465 & 350 & 17.1 \\
\hline
\end{tabular}

(AFM) results show that droplets appear with a density in the order of $10^{7} \mathrm{~cm}^{-2}$ as shown in Fig. 1(a) when the growth temperature is $364^{\circ} \mathrm{C}$. The big ones are about $1200 \mathrm{~nm}$ in diameter and $300 \mathrm{~nm}$ in height, while the small ones are about $700 \mathrm{~nm}$ in diameter and $180 \mathrm{~nm}$ in height. Energy dispersive X-ray (EDX) measurements using a scanning electron microscope (SEM) shows the relative percentages of In and $\mathrm{Bi}$ atoms to be $89.4 \%$ and $7.3 \%$, respectively, in the droplets (Fig. 1(d)), while the Bi concentration is very low between the droplets (Fig. 1(e)). A smooth surface could be obtained at $324^{\circ} \mathrm{C}$ with a low surface roughness value of only $0.93 \mathrm{~nm}$ from a $5 \times 5 \mu \mathrm{m}^{2}$ scan, see Fig. 1(b). If the growth temperature is further lowered to $275^{\circ} \mathrm{C}$, a whisker-like surface feature with a high roughness value of $17 \mathrm{~nm}$ is observed as shown in Fig. 1(c). The dependence of the morphology on the growth temperature will be discussed later.

Figure 2(a) shows high-resolution X-ray diffraction (XRD) (004) $\omega-2 \theta$ rocking curves for the three samples. The right narrow peak with a full width at half maximum (FWHM) of around 20 arcsec corresponds to the InP substrate and buffer, while the left peak corresponds to the InPBi layer. A weak and wide peak can be seen in InPBi grown at $364^{\circ} \mathrm{C}$. A narrow FWHM of 46 arcsec is obtained from the sample grown at $324^{\circ} \mathrm{C}$. Interference fringes can be clearly seen indicating a sharp InPBi/InP interface. The InPBi thickness is estimated to be about $420 \mathrm{~nm}$ from the simulations on the fringe separation. The strain relaxation is determined to be less than $5.0 \%$ from the two symmetric (004) scans and two asymmetric (115) scans and the lattice mismatch with respect to the InP substrate is $0.24 \%$ assuming the same Poisson ratio as InP. In order to confirm the single crystallization of the InPBi layer, the reciprocal space maps (RSM) of the $430 \mathrm{~nm}$ thick InPBi on both (004) and (115) planes were performed and plotted in logarithmic scale in Fig. 2(b) and 2(c). Two main peaks along with several satellite peaks can be clearly seen, in which the one with strong intensity belongs to the substrate, and the one with weak intensity belongs to the InPBi layer. The FWHM of the InP substrate peak and the InPBi is only 12.5 and 42.0 arcsec in (004) map, respectively, indicating excellent single crystal quality for a relatively thin epi-layer. The mismatch with respect to InP is $0.24 \%$ with strain relaxation of $5.4 \%$ which is the same as the value obtained using four $\omega-2 \theta$ scans. As the growth temperature is further lowered to $275^{\circ} \mathrm{C}$, the InPBi peak becomes broadened. This layer has a mismatch of $0.25 \%$ with respect to InP and the strain relaxation increases to $21 \%$. These results reveal that growth temperature is a critical parameter to obtain smooth InPBi single crystal.

After the growth temperature optimized, several samples with similar growth parameters of sample b were grown. The Bi concentration was determined by Rutherford Backscattering Spectroscopy (RBS), which is a powerful method to determine the atom concentration especially for heavy atoms since the $\mathrm{He}^{2+}$ beam will be easily scattered after impinging on them. A typical result is shown in Fig. 3. The Bi concentration is obtained by simulating the random spectrum. The black dotted line, the red dashed line and the blue line are the randomly oriented, the simulated and the aligned spectra, respectively. The surface spikes of the In signal at the channel number 455 of the [100] aligned curve is an artifact of the channeling analysis. Since the surface atoms are more exposed to the $\mathrm{He}^{2+}$ beam, they don't channel. From the RBS results, the Bi concentration is determined to be $2.4 \pm 0.4 \%$ with $94 \pm 5 \%$ Bi atoms at substitutional sites.

Figure 4(a) shows a typical cross-section Z-contrast scanning transmission electron microscope (STEM) image. A clear InPBi/ InP interface and a lightly wavy surface are observed. The InPBi layer thickness is measured to be $423 \pm 5 \mathrm{~nm}$, close to the thickness determined by the XRD interference fringes. From the high resolution TEM results (not shown here), the lattice arrangement in both InP buffer side and InPBi side is very regular. The result indicates the epitaxial relation and very high interface quality. Figures $4(\mathrm{~b})-(\mathrm{d})$
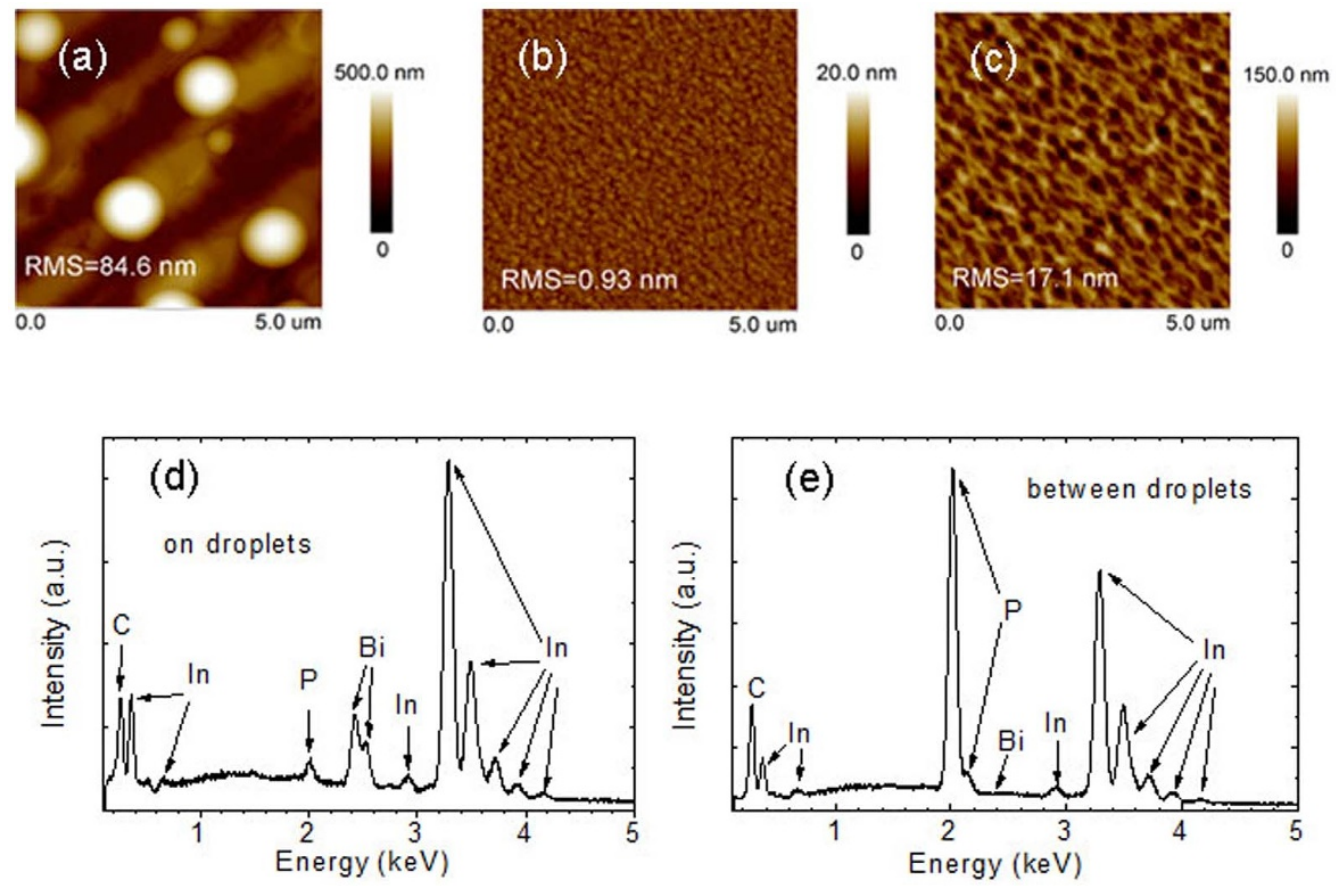

Figure $1 \mid$ (a), (b) and (c) show surface morphology of $5 \times 5 \mu \mathrm{m}^{2}$ InPBi measured by AFM using the tapping mode when grown at 364,324 and $275^{\circ} \mathrm{C}$, respectively. (d) and (e) show the EDX measurement results in an SEM from the InPBi sample grown at $364^{\circ} \mathrm{C}$. 

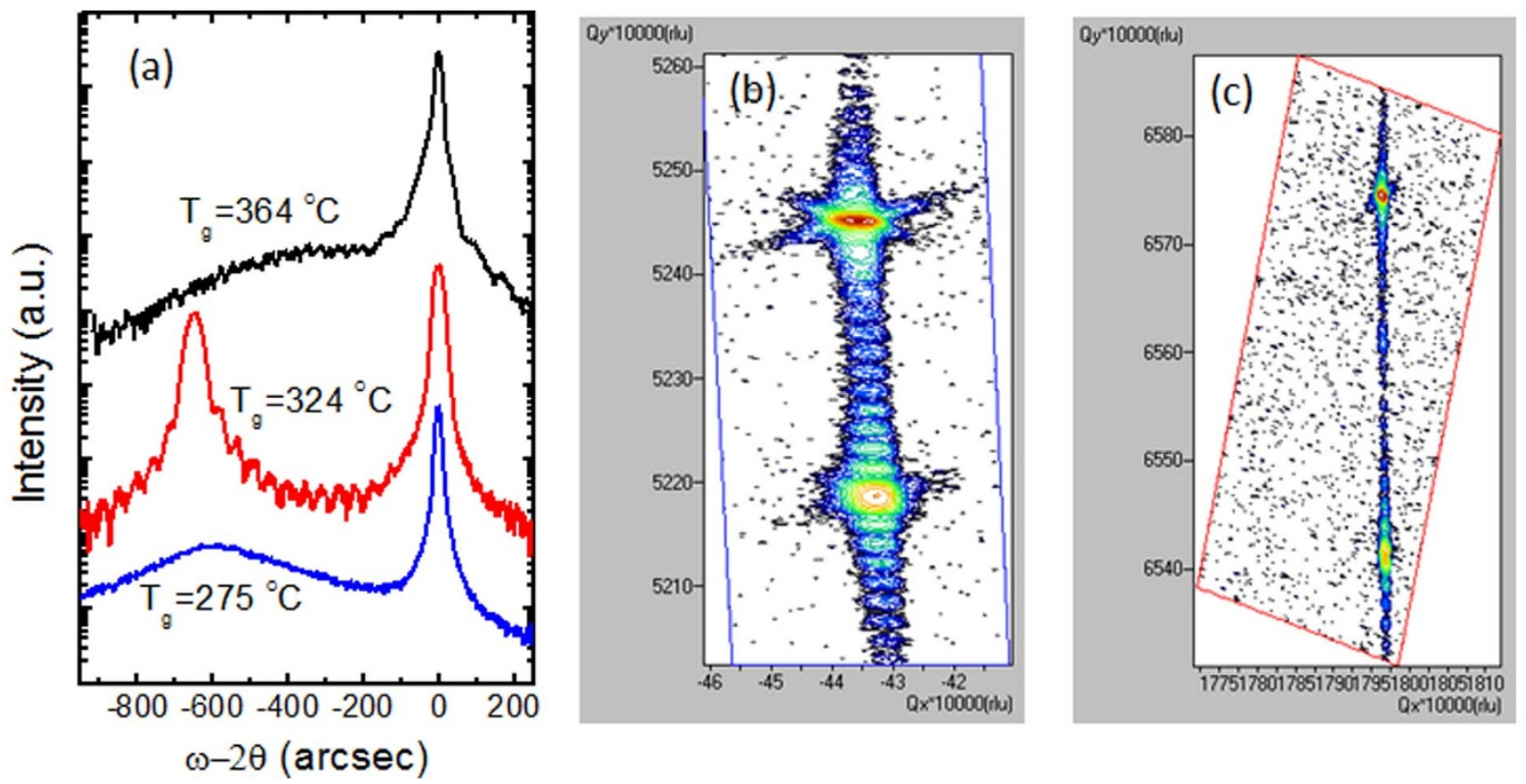

Figure $2 \mid$ (a) High resolution XRD (004) $\omega$-20 rocking curves from the InPBi samples. (b) and (c) are the reciprocal space maps of the 430 nm thick InPBi on both (004) and (115) planes, respectively.

show the EDX-mapping results in STEM for $\mathrm{Bi}$, In and $\mathrm{P}$, respectively. $\mathrm{Bi}$ incorporation in the InPBi layer is clearly revealed in quite uniform distribution within the probed resolution. We note, however, information on Bi clusters or non-uniformity within the $10 \mathrm{~nm}$ regime can't be resolved from such a mapping, as the measured data represent the average value for each element within the e-beam probe which is typically of $10 \mathrm{~nm}$. Nevertheless, we could not identify any detectable hint of Bi-related clusters from high-resolution TEM lattice imaging and high-resolution Z-contrast imaging, in contrast to our previous findings in GaAsBi samples ${ }^{15}$. For In and $\mathrm{P}$ mapping, the concentration of both elements is found to be reduced in InPBi

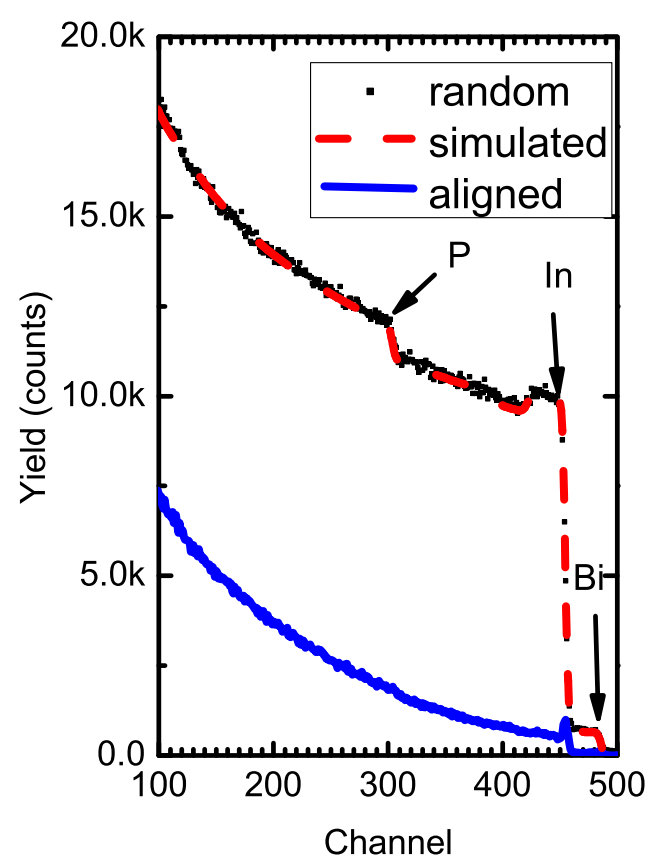

Figure 3 Randomly oriented (black dotted line), simulated (red dashed line) and aligned (blue line) RBS spectra for the InPBi layer grown at $324^{\circ} \mathrm{C}$. compared with in InP. This is more clearly visible by the steps in Fig. 3(e) where depth profiles of the average $\mathrm{Bi}$, In and $\mathrm{P}$ counts from EDX-mapping are shown. The detected drop of In concentration in InPBi is somewhat unexpected and can have important consequences for defect formations as will be discussed below.

Optical properties. Figure 5(a) shows PL spectra of sample b at 8$300 \mathrm{~K}$ and the $\mathrm{InP}$ reference samples at $300 \mathrm{~K}$, respectively. The InPBi sample at $300 \mathrm{~K}$ reveals strong and broad PL signals at 1.4$2.7 \mu \mathrm{m}$, centered at $1.9 \mu \mathrm{m}$ and its FWHM is as wide as around $660 \mathrm{~nm}(200 \mathrm{meV})$. The peak intensity is about 8 times stronger while the integrated PL intensity is almost 80 times higher compared with the InP reference sample. Careful analysis of the PL spectra reveals two peaks centered at $1.78 \mu \mathrm{m}(0.70 \mathrm{eV})$ and $2.04 \mu \mathrm{m}(0.61 \mathrm{eV})$ at $300 \mathrm{~K}$, respectively, using the Gaussian fitting, and three peaks at $\mathrm{T}<200 \mathrm{~K}$. This strong PL signal is not observed from the InP reference sample, where only an interband transition peak at $0.92 \mu \mathrm{m}(1.34 \mathrm{eV})$ can be seen. Figure 5(b) shows the normalized transmission spectra of the InPBi sample and InP reference sample of similar thickness at $300 \mathrm{~K}$. The small bump observed at $0.92 \mathrm{eV}$ is due to water absorption. The transmittance drops rapidly at about $1.3 \mathrm{eV}$ mainly due to the large absorption coefficient near the band gap of $\mathrm{InP}^{16}$. Compared with the InP reference sample, the transmittance of InPBi sample remains essentially the same for the photon energy below $1.0 \mathrm{eV}$, and decreases faster for the photon energy above $1.0 \mathrm{eV}$, indicating stronger optical absorption in this energy regime and a smaller band gap of InPBi. Using the plot of square of absorption coefficient vs energy (not shown here), the band gap of InPBi is deduced to be $1.23 \mathrm{eV}$, giving an estimate of band gap reduction of $46 \mathrm{meV} / \% \mathrm{Bi}$. Comparing absorption with PL spectra, we notice that there is no energy overlaps of the interested parts of the signals. The absorption spectra show near band gap features at above $1.0 \mathrm{eV}$ while the broad PL energy falls within $0.4-1.0 \mathrm{eV}$.

\section{Discussion}

To overcome the large miscibility gap and the weak bonding of III-Bi compared with those of conventional bonding of III-Vs, both the growth temperature and the V/Bi flux ratio should be kept low. This is the general strategy for almost all epitaxially grown dilute bismides. 

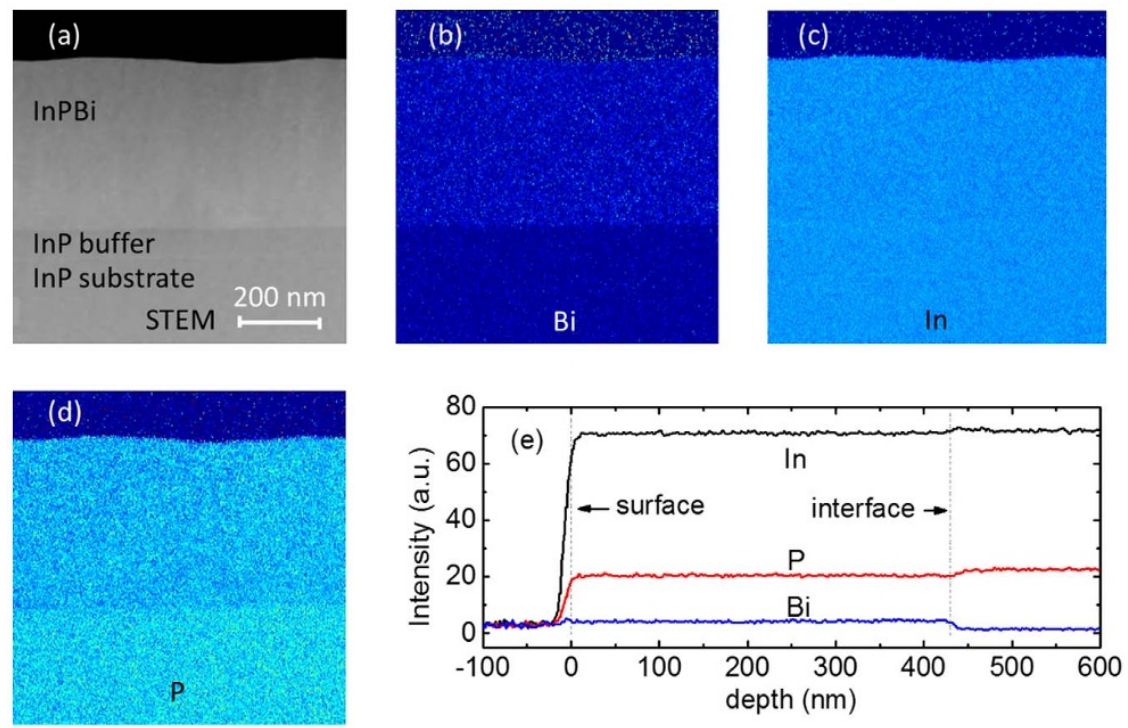

Figure $4 \mid$ (a) A typical STEM image and EDX-mapping results for (b) Bi, (c) In and (d) P. Figure (e) shows the depth profiles of the average Bi, In and P counts from the EDX-mapping (b)-(d).

In the case of InPBi, use of a low $\mathrm{P}$ flux has a risk of forming In droplets. For the InPBi grown at $364^{\circ} \mathrm{C}$, large metallic droplets are observed by AFM. However, the InP thin film grown under the same conditions reveals a smooth surface. This indicates that the $\mathrm{P}$ flux is
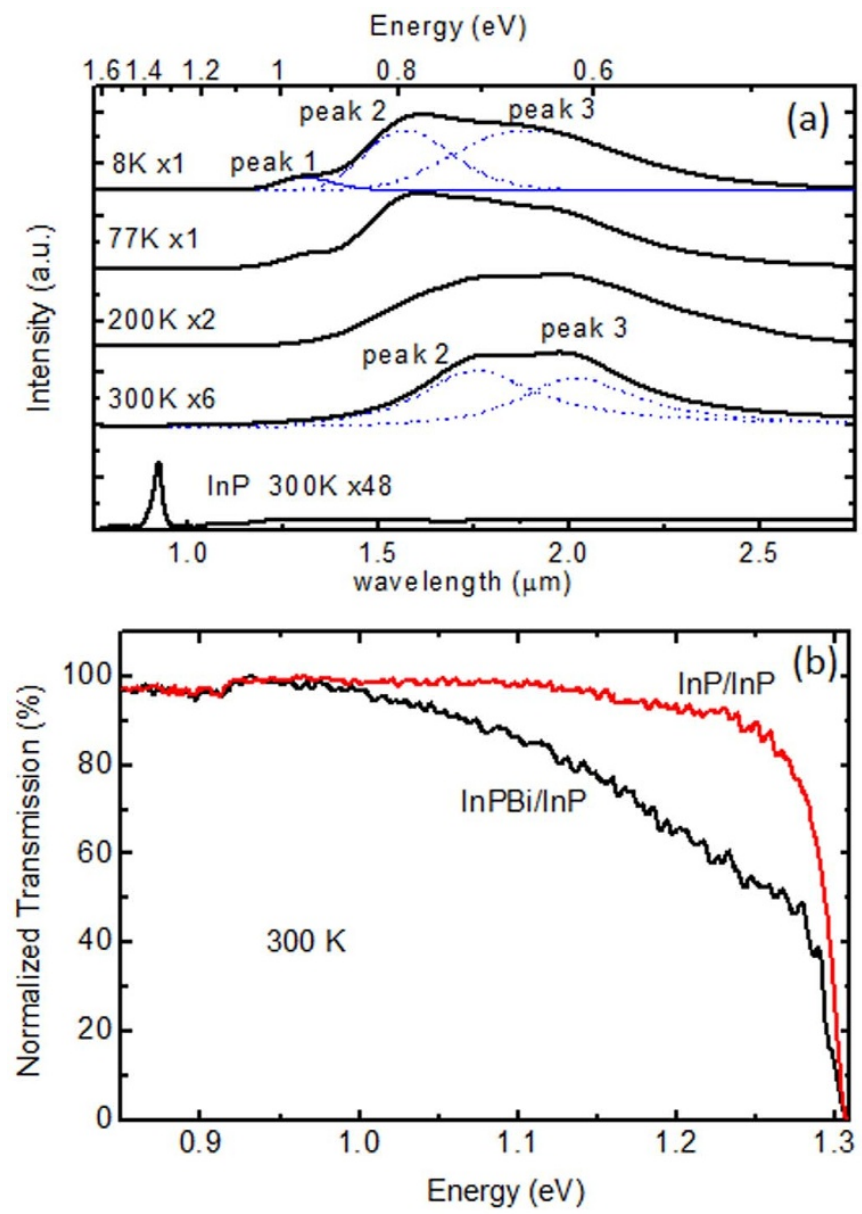

Figure $5 \mid$ (a) PL spectra of the InPBi sample measured at 8-300 K and the InP reference sample at $300 \mathrm{~K}$. The dashed blue lines are the Gaussian peak fitting. (b) The normalized transmission spectra of the InPBi sample and the InP reference sample at $300 \mathrm{~K}$. high enough to ensure a P-rich surface reconstruction and thus a smooth surface. When $\mathrm{Bi}$ is added, large droplets are formed and the EDX measurement in SEM shows considerable amount of In in addition to Bi found in the droplets. This suggests that In is subject to alloying with $\mathrm{Bi}$. It should be noted that both In and $\mathrm{Bi}$ will be in liquid form when the growth temperature is higher than their melting points $\left(156.6^{\circ} \mathrm{C}\right.$ and $271^{\circ} \mathrm{C}$ for In and $\mathrm{Bi}$, respectively) and there indeed exist several In-Bi phases in the phase diagram ${ }^{17}$. The weak bonding nature of $\mathrm{Bi}$ atoms renders themselves to accumulate on the InP surface. Formation of In-Bi alloys consumes both In and $\mathrm{Bi}$ atoms and hinders the InP growth.

When the growth temperature is reduced to $324^{\circ} \mathrm{C}$ measured by the thermocouple, high quality InPBi thin film is demonstrated. Although the real growth temperature is unknown, we believe it will be quite close to the $\mathrm{Bi}$ melting point. The probability of $\mathrm{Bi}$ atoms sticking on surface and incorporation into lattice are enhanced when decreasing the growth temperature. When the impingent $\mathrm{Bi}$ atoms are balanced by their incorporation to lattice sites, single crystal InPBi film is achieved and no excess $\mathrm{Bi}$ atoms will form Bi droplets on surface. Bismuth is known to be as an excellent surfactant, which ensures good surface and interface quality.

When the growth temperature is further lowered, excess $\mathrm{Bi}$ and $\mathrm{P}$ atoms will accumulate on InP. Meanwhile, $\mathrm{PH}_{3}$ decomposition rate decreases with reducing growth temperature in gas source $\mathrm{MBE}$ and the resulting InPBi growth dynamics becomes rather complicated. The reference InP sample grown at $275^{\circ} \mathrm{C}$ with a slightly lower $\mathrm{PH}_{3}$ pressure of 320 Torr shows a similar surface feature as that of InPBi shown in Fig. 1(c). Further study is necessary to account for the observed surface morphology.

From the (004) XRD results shown in Fig. 2(a), there is some Bi incorporated in the sample grown at $364^{\circ} \mathrm{C}$. As the rocking-curve signal is broad and weak, it is difficult to estimate the Bi content. The sample grown at $324^{\circ} \mathrm{C}$ reveals nice pendellösung fringes in the (004) scan indicating good interface. If we assume the Vegard's law is valid, the extrapolated lattice constant of zinc-blende InBi will be $6.52 \pm$ $0.11 \AA$ which is very close to the previously reported value of $6.5 \AA^{18}$. The measured lattice mismatch is $0.24 \%$ for InPBi grown at $324^{\circ} \mathrm{C}$.

The PL measurement reveals unexpectedly strong and broad signals at $0.4-1.0 \mathrm{eV}$ at $300 \mathrm{~K}$ which are absent in the reference $\mathrm{InP}$ thin film grown at the same growth conditions. The absorption spectra show no difference between InPBi and InP for energy below $1.0 \mathrm{eV}$ but enhanced absorption in InPBi for energy above $1.0 \mathrm{eV}$. This 
implies that physical origins can be different in the two energy regimes. Previous spectroscopic studies of InP:Bi grown by liquid phase epitaxy with the Bi concentration in the doping level of 10100 ppm show many spectral features within $100 \mathrm{meV}$ below the InP band gap at low temperatures, but no signals in the mid-gap at room temperature ${ }^{3,4}$. In these materials, $\mathrm{Bi}$ can be regarded as dopant and modelled by a resonant defect level within the energy band structure of the host matrix. In our case, the Bi concentration is much higher, and $\mathrm{Bi}$ interaction as well as cluster formation must be considered. There have been several theoretical investigations on the band structures of $\mathrm{GaAsBi}^{19-23}$ and $\mathrm{GaPBi}^{23}$ to account for the band gap reduction of dilute bismides, but the physical explanation of whether it is due to the band anti-crossing or just the alloying and disorder is still under debate ${ }^{23}$. It is theoretically predicted that the Bi-related defect resonant level lies $80 \mathrm{meV}$ below the valence band of $\mathrm{GaAs}^{22}$ and $122 \mathrm{meV}$ above the valence band of $\mathrm{GaP}^{23}$. For disordered $\mathrm{GaP}_{1}-{ }_{\mathrm{x}} \mathrm{Bi}_{\mathrm{x}}$ including clustering effect with $x$ up to $4.88 \%$, broad band tail states exist at about $0.15-0.5 \mathrm{eV}$ above the valence band $\mathrm{d}^{23}$. If we assume similar band tail states also exist in InPBi, this may explain the near band gap features observed in the absorption measurement. However, it gives low energy bound of $0.84 \mathrm{eV}$ (the band gap of InP is $1.34 \mathrm{eV}$ at $300 \mathrm{~K}$ ), still larger than the observed $0.70 \mathrm{eV}$ and $0.61 \mathrm{eV}$ in PL at $300 \mathrm{~K}$. On the other hand, if the PL signals were due to the band anticrossing, this would give exceptionally large band gap bowing of $267 \mathrm{meV} / \% \mathrm{Bi}$. Therefore, current existing theory can't explain the PL signals at the mid band gap range. Reihlen et al. reported large energy differences of 70-200 $\mathrm{meV}$ between PL and absorption at $300 \mathrm{~K}$ in $\mathrm{InP}_{\mathrm{x}} \mathrm{Sb}_{1-{ }_{\mathrm{x}}}$ for $0.1<x<0.4^{24}$. They attributed this difference to the combination of deep centers and intrinsic band tail recombination which were indistinguishable from the spectroscopy. The large band tail was believed to result from the compositional clustering. The compositional inhomogeneity usually induces localized states and causes S-shape in temperature dependent PL peak energy, often found in dilute nitrides ${ }^{25}$. However, in the temperature dependent PL spectra shown in Fig. 4(a), we don't observe any noticeable S-shape curves.

We tentatively attribute the strong and broad PL signals at $300 \mathrm{~K}$ to deep level luminescence. The deep levels can be $\mathrm{Bi}_{\text {In }}$ anti-sites, $\mathrm{P}$ and/or In vacancies or the above combinations. The EDX-mapping results shown in Fig. 3 unambiguously confirm the decreases of both In and $\mathrm{P}$ atoms in InPBi compared with in InP. This gives some evidence that In and $\mathrm{P}$ vacancies may exist. Indeed, the scanning tunneling microscope (STM) on cross-sectional InPBi confirms the existence of $\mathrm{P}$ vacancies (not shown here). Bi atoms are generally believed to occupy $\mathrm{P}$ sites in dilute bismide. However, this will introduce very high local strain in InPBi. Our first principle calculations show that the overall formation energy is smaller for Bi taking In lattice positions instead of $\mathrm{P}$ positions. Also Bi can be of $3+$ valence due to the relativistic effect of the two $6 \mathrm{~s}$ electrons. These suggest that $\mathrm{Bi}_{\mathrm{In}}$ anti-site is physically favorable and its concentration can be quite high. More experimental works, such as deep level transient spectroscopy (DLTS) and time resolved PL, are necessary to clarify the nature of those deep levels and carrier dynamics. Likewise, new theoretical model or simulations are stimulated to give physical explanations of the observed PL spectra.

In summary, new InPBi single crystal has been successfully grown by gas source $\mathrm{MBE}$ for the first time. High surface and structural quality has been demonstrated and confirmed by AFM, XRD and TEM measurement. RBS measurement shows that the Bi concentration is $2.4 \pm 0.4 \%$, with $94 \pm 5 \% \mathrm{Bi}$ atoms at substitutional sites. Room temperature PL measurement reveals a very broad and strong spectrum from 1.4 to $2.7 \mu \mathrm{m}$ while the optical absorption shows a band gap reduction of $110 \mathrm{meV}$ with respect to InP. The observed PL spectrum is likely from deep levels and can't be explained by the existing theory.

\section{Methods}

Molecular Beam Epitaxy. Samples were grown on (100) semi-insulating InP substrates by V90 gas source MBE. $\mathrm{P}_{2}$ was cracked from phosphine at $1000^{\circ} \mathrm{C}$ and its flux was controlled by regulating the gas pressure in the gas line. Elementary In and $\mathrm{Bi}$ sources were used and their fluxes were controlled by adjusting the effusion cell temperatures. Both substrate and cell temperature were measured by thermocouples.

Prior to growth, oxide desorption from the substrate surface was carried out by heating the substrate with a $\mathrm{P}_{2}$ flux impingement on the surface. The complete desorption of the oxide layer was determined using the reflection high energy electron diffraction (RHEED), by a sharp transition to $(2 \times 4)$ pattern at about $524^{\circ} \mathrm{C}$. A $75 \mathrm{~nm}$ un-doped InP buffer layer was grown at $474^{\circ} \mathrm{C}$ with the $\mathrm{PH}_{3}$ pressure of 630 Torr to improve the surface quality. $420 \mathrm{~nm}$ thick $\mathrm{InP}_{1}-{ }_{\mathrm{x}} \mathrm{Bi}_{\mathrm{x}}$ thin films were then grown at $275-364^{\circ} \mathrm{C}$ at $0.86 \mu \mathrm{m} / \mathrm{h}$ with a low $\mathrm{P}$ flux to facilitate the Bi incorporation into the lattice. InP reference samples were grown with otherwise the same growth conditions as those for $\mathrm{InP}_{1}-{ }_{\mathrm{x}} \mathrm{Bi}_{\mathrm{x}}$ for comparison.

Measurements. The surface morphology was measured by Bruker Icon atomic force microscopy (AFM) using the tapping mode. The scan area was $5 \times 5 \mu^{2}$. The electron acceleration voltage for the EDX measurement in SEM was set at $10 \mathrm{kV}$ to obtain good spatial resolution. Conventional X-ray diffraction $\omega-2 \theta$ scan was performed using a Philips X-pert high-resolution diffractometer to measure structural properties. The $\mathrm{Cu} \mathrm{K} \alpha 1$ wavelength was selected by a Ge (220) fourfold monochrometer. The STEM and the EDX-mapping measurements were performed on a FEI Tecnai G2 F20 microscope. Cross-section STEM specimens were prepared by focus ions beam. In the RBS measurement, a $2.275 \mathrm{MeV}^{4} \mathrm{He}^{2+}$ source was used to impinge on the sample surface. The PL and transmission spectra were measured using a Nicolet Megna 860 Fourier transform infrared (FTIR) spectrometer, in which a liquid-nitrogen cooled InSb detector and $\mathrm{a} \mathrm{CaF}_{2}$ beam splitter were used. A diodepumped solid state laser $(\lambda=514.5 \mathrm{~nm})$ was used as an excitation source for PL measurement.

1. Thomas, D. G., Hopfield, J. J. \& Frosch, C. J. Isoelectronic Traps Due to Nitrogen in Gallium Phosphide. Phys Rev Lett 15, 857-860 (1965).

2. Faulkner, R. \& Dean, P. Electronic structure of ground and excited states of isoelectronic traps. J Lumin 1, 552-561 (1970).

3. Dean, P. J., White, A. M., Williams, E. W. \& Astles, M. G. The isoelectronic trap bismuth in indium phosphide. Solid State Commun 9, 1555-1558 (1971).

4. Ruhle, W. et al. Isoelectronic Impurity States in Direct-Gap III-V Compounds: The case of InP: Bi. Phys Rev B 18, 7022-7032 (1978).

5. Tisch, U., Finkman, E. \& Salzman, J. The anomalous bandgap bowing in GaAsN. Appl Phys Lett 81, 463-465 (2002).

6. Weyers, M., Sato, M. \& Ando, H. Red shift of photoluminescence and absorption in dilute GaAsN alloy layers. Jpn J Appl Phys 31, L853-L855 (1992).

7. Francoeur, S. et al. Band gap of $\mathrm{GaAs}_{1-{ }_{\mathrm{x}}} \mathrm{Bi}_{\mathrm{x}}, 0<\mathrm{x}<3.6 \%$. Appl Phys Lett 82, 3874-3876 (2003).

8. Alberi, K. et al. Valence-band anticrossing in mismatched III-V semiconductor alloys. Phys Rev B 75, 045203 (2007).

9. Gustavsson, J., Wei, Y. Q., Sadeghi, M., Wang, S. \& Larsson, A. 10 Gbit/s modulation of $1.3 \mu \mathrm{m} \mathrm{GaInNAs} \mathrm{lasers} \mathrm{up} \mathrm{to} 110^{\circ} \mathrm{C}$. Electron Lett 42, 925-926 (2006).

10. Wiemer, M., Sabnis, V. \& Yuen, H. High and Low Concentrator Systems for Solar Electric Applications VI, 8108, 810804-810805 (2011)

11. Berding, M. A., Sher, A., Chen, A. B. \& Miller, W. E. Structural properties of bismuth-bearing semiconductor alloys. J Appl Phys 63, 107-115 (1988).

12. Dominguez, L. et al. Formation of Tetragonal InBi Clusters in InAsBi/InAs (100) Heterostructures Grown by Molecular Beam Epitaxy. Appl Phys Express 6, 112601 (2013).

13. Oe, K., Ando, S. \& Sugiyama, K. InSb $1-{ }_{x} \mathrm{Bi}_{\mathrm{x}}$ Films Grown by Molecular Beam Epitaxy. Jpn J Appl Phys 20, L303-L306 (1981).

14. Noreika, A. J., Takei, W. J., Francombe, M. H. \& Wood, C. E. C. Indium antimonide-bismuth compositions grown by molecular beam epitaxy. J Appl Phys 53, 4932-4937 (1982)

15. Wu, M., Luna, E., Puustinen, J., Guina, M. \& Trampert, A. Formation and phase transformation of Bi-containing QD-like clusters in annealed GaAsBi. Nanotechnol in press (2014).

16. Turner, W., Reese, W. \& Pettit, G. Exciton absorption and emission in InP. Phys Rev 136, A1467 (1964).

17. Cui, Y. et al. Thermodynamic calculation of phase diagram in the Bi-In-Sb ternary system. Mater Trans 43, 1879-1886 (2002).

18. Okamoto, H. \& Oe, K. Growth of metastable alloy InAsBi by low-pressure MOVPE. Jpn J Appl Phys 37, 1608-1613 (1998).

19. Alberi, K. et al. Valence band anticrossing in $\mathrm{GaBi}_{\mathrm{x}} \mathrm{As}_{1}-\mathrm{x}$. Appl Phys Lett 91, 051909-051903 (2007)

20. Deng, H. et al. Band crossing in isovalent semiconductor alloys with large size mismatch: First-principles calculations of the electronic structure of $\mathrm{Bi}$ and $\mathrm{N}$ incorporated GaAs. Phys Rev B 82, 193204 (2010).

21. Janotti, A., Wei, S. \& Zhang, S. Theoretical study of the effects of isovalent coalloying of Bi and N in GaAs. Phys Rev B 65, 115203 (2002).

22. Zhang, Y., Mascarenhas, A. \& Wang, L. Similar and dissimilar aspects of III-V semiconductors containing Bi versus N. Phys Rev B 71, 155201 (2005). 
23. Usman, M., Broderick, C. A., Lindsay, A. \& O’Reilly, E. P. Tight-binding analysis of the electronic structure of dilute bismide alloys of GaP and GaAs. Phys Rev B 84, 245202 (2011).

24. Reihlen, E., Jou, M., Fang, Z. \& Stringfellow, G. Optical absorption and emission of InPSb alloys. J Appl Phys 68, 4604 (1990).

25. Grenouillet, L. et al. Evidence of strong carrier localization below $100 \mathrm{~K}$ in a GaInNAs/GaAs single quantum well. Appl Phys Lett 76, 2241-2243 (2000).

\section{Acknowledgments}

The authors wish to acknowledge the financial support of the National Basic Research Program of China (Grant No. 2014CB643902), the Key Program of Natural Science Foundation of China (Grant No. 61334004), the "Strategic Priority Research Program" of the Chinese Academy of Sciences (Grant No. XDA5-1), the Key Research Program of the Chinese Academy of Sciences (Grant No. KGZD-EW-804) and the Creative Research Group Project of Natural Science Foundation of China (Grant No. 61321492).

\section{Author contributions}

S.M.W. conceived the idea behind this work, proposed the preliminary explanation for PL results and revised the manuscript. K.W. developed and grew all the samples, wrote the first version, performed the AFM and Raman measurement. Y.G. performed the PL and transmission measurement, revised the manuscript. C.Z.K. assisted in the sample growth. H.F.Z., L.Y.Z. and W.W.P. assisted in various material characterization measurements. M.J.W. performed the TEM measurement. P.F.L. performed the first principle calculations. Q.G. and all the other authors were involved in the discussion.

\section{Additional information}

Competing financial interests: The authors declare no competing financial interests. How to cite this article: Wang, K. et al. InPBi Single Crystals Grown by Molecular Beam Epitaxy. Sci. Rep. 4, 5449; DOI:10.1038/srep05449 (2014).

This work is licensed under a Creative Commons Attribution-NonCommercialNoDerivs 4.0 International License. The images or other third party material in this article are included in the article's Creative Commons license, unless indicated otherwise in the credit line; if the material is not included under the Creative Commons license, users will need to obtain permission from the license holder in order to reproduce the material. To view a copy of this license, visit http:// creativecommons.org/licenses/by-nc-nd/4.0/ 